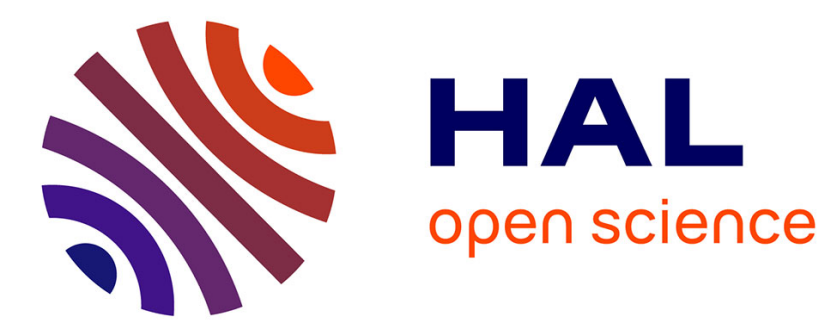

\title{
Problèmes posés par la réalisation des filtres interférentiels utilisés dans les démultiplexeurs optiques
}

\author{
B. Bovard, A. Fornier, E. Pelletier, R. Richier
}

\section{To cite this version:}

B. Bovard, A. Fornier, E. Pelletier, R. Richier. Problèmes posés par la réalisation des filtres interférentiels utilisés dans les démultiplexeurs optiques. Revue de Physique Appliquée, 1984, 19 (2), pp.103-105. 10.1051/rphysap:01984001902010300 . jpa-00245158

\section{HAL Id: jpa-00245158 https://hal.science/jpa-00245158}

Submitted on 1 Jan 1984

HAL is a multi-disciplinary open access archive for the deposit and dissemination of scientific research documents, whether they are published or not. The documents may come from teaching and research institutions in France or abroad, or from public or private research centers.
L'archive ouverte pluridisciplinaire HAL, est destinée au dépôt et à la diffusion de documents scientifiques de niveau recherche, publiés ou non, émanant des établissements d'enseignement et de recherche français ou étrangers, des laboratoires publics ou privés. 


\title{
Problèmes posés par la réalisation des filtres interférentiels utilisés dans les démultiplexeurs optiques
}

\author{
B. Bovard, A. Fornier, E. Pelletier et R. Richier \\ Centre d'Etude des Couches Minces (associé au CNRS), Ecole Nationale Supérieure de Physique de Marseille, \\ Domaine Universitaire de Saint-Jérôme, 13397 Marseille Cedex 13, France
}

(Reçu le 3 juin 1983, révisé le 17 novembre, accepté le 18 novembre 1983)

\begin{abstract}
Résumé. - Pour réaliser des multi-démultiplexeurs de hautes performances, on est conduit à employer des filtres Fabry-Pérot multicavités. On montre comment les problèmes de réalisations de ces filtres ont été résolus. C'est en particulier grâce à un contrôle optique direct de la formation de l'empilement que l'on peut garantir un bon accord entre les propriétés optiques attendues et celles effectivement obtenues.
\end{abstract}

\begin{abstract}
The problems of wavelength selective optical coatings suitable for multiplexing with large cross-talk attenuations lead to the construction of square band-pass filtres. A design of a multilayer stack is proposed and we analyse the principal problems of production especially the optical monitoring during evaporation. The agreement between theoretical and experimental performances is good.
\end{abstract}

Le multi ou démultiplexage optique des différents canaux utilisés en télécommunication par fibre optique peut être assuré par des dispositifs utilisant des réseaux de diffraction, des miroirs dichroïques ou des filtres interférentiels passe-bande.

Dans ce dernier cas, les caractéristiques des multidémultiplexeurs (largeur de la bande passante, transparence, taux de réjection, ...) conduisent à envisager l'emploi de filtres Fabry-Perot multicavités.

\section{Choix de la formule. Propriétés de l'empilement.}

1.1 ChOIX De LA FORMULE. - L'expérience et les calculs de synthèse conduisent à des filtres F.P. triples de type :

$$
\left(M_{4} 2 \mathrm{H} \mathrm{M}_{4}\right) \mathrm{B}\left(\mathrm{M}_{4} 2 \mathrm{H} \mathrm{M}_{4}\right) \mathrm{B}\left(\mathrm{M}_{4} 2 \mathrm{H} \mathrm{M}_{4}\right)
$$

$M_{4}=$ HBHB $\quad H=$ couche haut indice d'épaisseur optique $\lambda_{0} / 4$.

B = couche bas indice d'épaisseur optique $\lambda_{0} / 4$

Les caractéristiques de ce filtre sont :

$T \max =1$

bande passante à $3 \mathrm{~dB}=21,6 \mathrm{~nm}$. Figure 1 (1)

Taux de réjection à $\lambda_{0} \mp 35 \mathrm{~nm}=35 \mathrm{db}$.

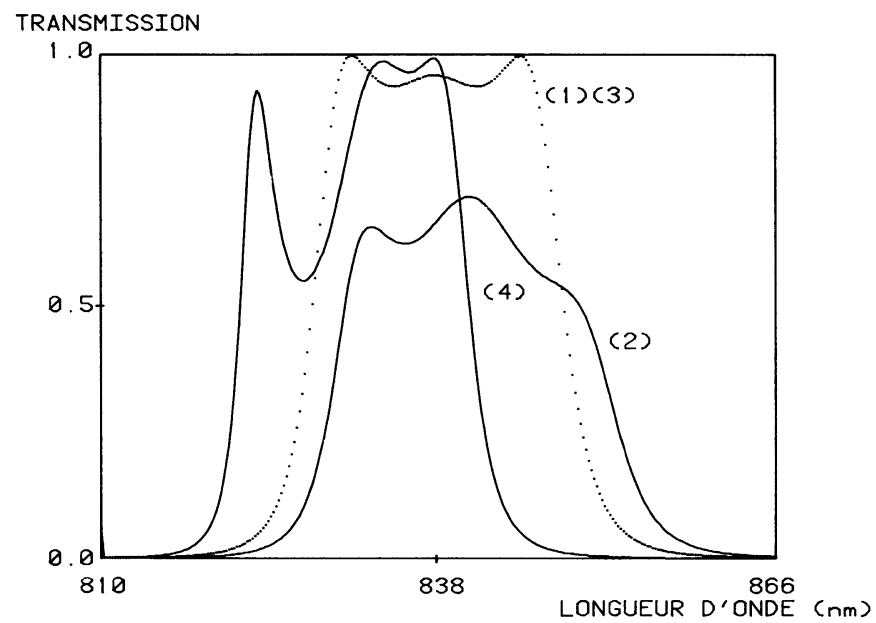

Fig. 1. - Influence des erreurs d'épaisseur sur le profil spectral du filtre F.P. triple cavités.

[Effect of thickness errors on the optical transmittance of a THW filtre.]

1.2 INFLUENCE DES ERREURS D'ÉPAISSEUR. - La réalisation de tels filtres ne saurait s'effectuer sans erreurs, dont on peut définir deux grandes classes :

1.2.1 Erreurs statiques. - Leur valeur reste constante mais leur effet varie considérablement avec la méthode de contrôle envisagée : 
Si le dépôt de la couche de rang $i$ est indépendant des erreurs commises sur les $i-1$ couches précédentes on peut obtenir un profil spectral tel que celui de la figure 1 (2). Dans ce cas toutes les épaisseurs sont supposées parfaites à part la $25^{\mathrm{e}}$ couche dont l'épaisseur est augmentée de $5 \%$. On rencontre ces conditions lors de l'utilisation d'une balance à quartz ou d'un contrôle optique sur des témoins successifs [1].

$\mathrm{Si}$ au contraire on utilise un contrôle optique direct de tout l'empilement sur l'échantillon lui-même, alors le dépôt de la couche de rang $i$ est conditionné par toute l'histoire antérieure de l'empilement. Dans ce cas on a montré [2] que l'on bénéficiait d'une compensation automatique des erreurs. Figure 1 (3) : le profil spectral est identique au profil théorique (Fig. 1 (1)). C'est le type de contrôle que l'on devra utiliser.

1.2.2 Erreurs dynamiques. - Elles évoluent dans le temps et proviennent, par exemple, de variation de température pendant le dépôt, de modification de l'indice de réfraction par adsorption d'eau lors du retour à la pression atmosphérique, etc. Dans ce cas il n'y a pas de compensation possible et on ne peut qu'étudier les causes d'erreurs pour les éviter ou les limiter.

\section{Contrôle des dépôts. Résultats.}

\subsection{MÉTHOdE DE CONTRÔLE.}

2.1.1 Principe. - Les empilements étant constitués de couches quart d'onde, on sait que le contrôle de l'épaisseur des couches s'effectue en détectant les extréma de transmission ou de réflexion pour la longueur d'onde choisie. On utilise pour cela les annulations de la dérivée de la transmission par rapport au temps [3]. Même dans ces conditions le succès n'est pas garanti et la figure 1 (4) montre le profil spectral obtenu en supposant une incertitude aléatoire comprise entre $10^{-4}$ et $5 \times 10^{-5}$ sur la détection des zéros.

2.1.2 Dispositif expérimental. - Pour atteindre ce degré de précision nous avons mis au point un système de contrôle essentiellement basé sur l'utilisation d'un spectromètre multi-canal associé à un calculateur P.D.P. 11 V. 03.

2.2 Résultat. - Avec du SZn comme matériau haut indice et de la cryolithe on a obtenu des filtres dont les propriétés sont très voisines des propriétés théoriques.

Figure 2 (1) : Profil spectral relevé sous vide.

Figure 2 (2) : Profil spectral théorique.

\section{Utilisation de matériaux résistants.}

Si l'on désire s'affranchir du collage d'un verre protecteur et obtenir une meilleure stabilité dans le

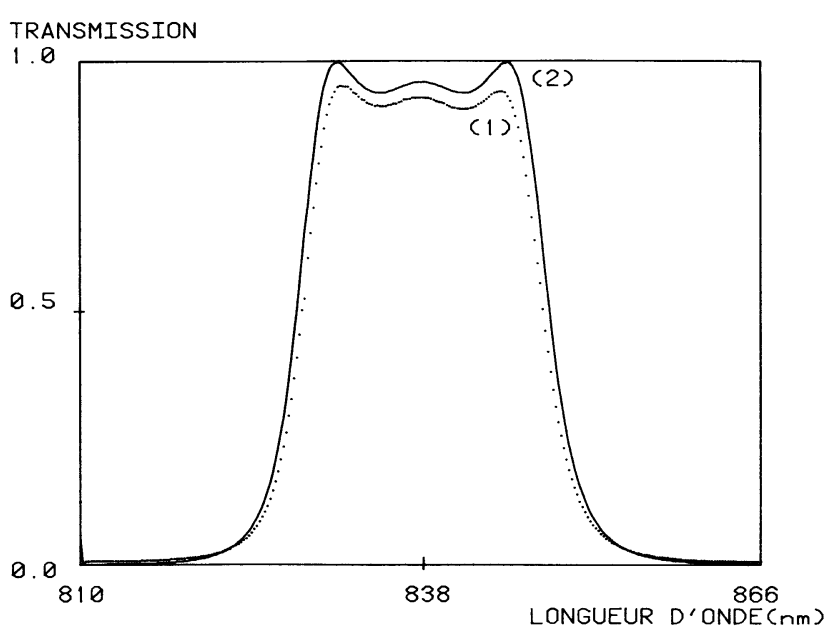

Fig. 2. - Profils spectraux calculé et expérimental d'un filtre Fabry-Pérot triple cavités.

[Recorded and calculated optical transmittance of a THW filtre.]

temps, des propriétés optiques, il faut envisager l'emploi de matériaux comme $\mathrm{TiO}_{2}, \mathrm{ZrO}_{2}, \mathrm{SiO}_{2}, \mathrm{MgF}_{2}, \ldots$ Tous ces matériaux condensent sur des substrats portés à 300 ou $400^{\circ} \mathrm{C}$ et, en ce qui concerne les

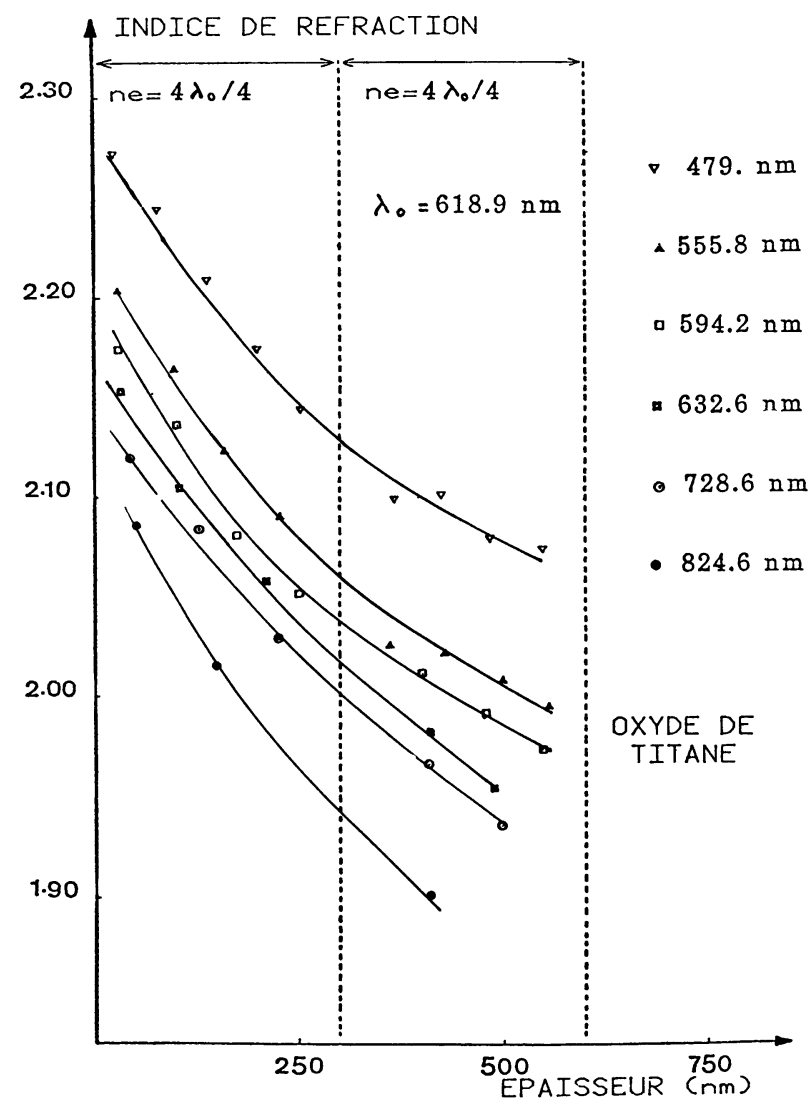

Fig. 3. - Valeurs mesurées in situ de l'indice de réfraction du $\mathrm{TiO}_{2}$ en fonction de l'épaisseur pour 6 longueurs d'onde.

[Measurements recorded during the deposition values for different wavelengths of the refractive index of $\mathrm{TiO}_{2}$ as a function of thickness.] 
oxydes métalliques, demandent un contrôle de l'oxydation pendant le dépôt. De nouveaux problèmes se posent alors :

Effet de température : on imagine facilement l'importance de la contraction des couches lors du retour à température ordinaire. Dans le meilleur des cas on assistera à une dérive de la longueur d'onde de centrage vers les courtes longueurs d'onde.

Effet de l'oxydation : rien ne permet de savoir à la fin du dépôt d'une couche si l'oxydation du matériau est complète. Ainsi lors du retour à la pression atmosphérique la stœechiométrie du matériau, donc son indice, peut varier.

Effet de structure : la structure lacunaire des couches entraîne des effets indésirables dont il faut tenir compte [4].

Adsorption d'eau lors du retour à la pression atmosphérique et par suite modification de l'indice de réfraction apparent [5].
Variation de l'indice de réfraction en fonction de l'épaisseur déposée d'où apparition d'un gradient d'indice à l'intérieur de la couche (Fig. 3).

Les moyens d'étude in situ dont le laboratoire dispose permettent de suivre l'évolution de l'épaisseur et de l'indice des matériaux pendant la formation de l'empilement et lors du retour à la pression atmosphérique.

A partir de ces études nous avons pu réaliser des filtres F.P. monocavité de quelques dizaines d'angströms de large dont la longueur d'onde de centrage dans les conditions d'utilisation, ne différait que de quelques $\AA$ de la valeur attendue.

Ainsi, en ce qui concerne les F.P. multicavités réalisés à partir de matériaux peu résistants et protégés il ne semble pas subsister de problèmes majeurs. Dans le cas des empilements de couches "dures" nous pensons être en mesure de réaliser des F.P. multicavités dans un avenir proche.

\section{Bibliographie}

[1] MaCleod, H. A., Thin Film Optical Filters (Adam Hilger) 1969.

Heather, M., Liddell, Computer Aided Techniques for the design of multilayer filters (Adam Hilger) 1981.

[2] Macleod, H. A., Opt. Acta 19 (1972) 1.

Bousquet, P., Fornier, A., Kowalczyk, R., Pelletier, E., Roche, P., Thin Solid Films 13 (1972) 295.

Macleod, H. A., Thin Solid Films 34 (1976) 335-342.

[3] Bousquet, P., Pelletier, E., Thin Solid Films 77 (1981) 165-179.
[4] Flory, F., Schmitt, B., Pelletier, E. and Macleod, H. A., S.P.I.E. Thin Film technology, April 18-22, 1983 Geneva, à paraître 1983.

[5] Ogura, S. and Macleod, H. A., Thin Solid Films 34 (1976) 371-375.

Macleod, H. A. and Richmond, D., Thin Solid Films 37 (1976) 163-169. 